\title{
Microwave-assisted synthesis of novel 2-naphthol bis-Mannich Bases
}

\author{
Po-Jung J. Huang, ${ }^{a}$ D. Youssef, ${ }^{\text {a,b }}$ T. Stanley Cameron, ${ }^{\mathrm{c}}$ and Amitabh Jha ${ }^{\mathrm{a}, *}$ \\ ${ }^{a}$ Department of Chemistry, Acadia University, Wolfville, B4P 2R6, Nova Scotia, Canada \\ ${ }^{b}$ Département des Sciences, Université Sainte-Anne, Church Point, NS, BOW 1M0, Canada \\ ${ }^{c}$ Department of Chemistry, Dalhousie University, Halifax, B3H 4J3, Nova Scotia, Canada \\ E-mail: amitabh.jha@acadiau.ca
}

\begin{abstract}
Mannich bases of 2-naphthol have the ability to chelate strongly to metal ions. Hence, they have great potential to be used as chiral catalysts, metallo-enzyme inhibitors and/or scavenger of heavy metal poisons. This paper deals with an efficient and expeditious microwave assistedsynthesis of novel bis-Mannich bases of 2-naphthols derived from aromatic aldehydes and diamines namely piperazine and $N, N$ '-dialkylethylenediamines under solvent-free conditions. These compounds were also prepared under conventional reflux in ethanol. The compounds of this series displayed interesting NMR behaviour.
\end{abstract}

Keywords: 2-Naphthol bis-Mannich bases, microwave, 2-naphthol, Mannich reaction, NMR spectroscopy, X-ray crystallography

\section{Introduction}

Mannich bases find variety of commercial applications. It was estimated that at least 35\% of Mannich bases related articles are published in pharmaceutical journals. They are known for their use in polymers, resins, surface active agents, ${ }^{1}$ detergent additives, ${ }^{2}$ and antioxidants. ${ }^{3}$ They have a broad range of biological activities including diuretic, ${ }^{4}$ antipsychotic, ${ }^{5}$ oxytocic, ${ }^{6}$ anticonvulsant, ${ }^{7}$ centrally acting muscle relaxant, ${ }^{8}$ antimalarial, ${ }^{9,10}$ antiviral $^{11}$ and anticancer. ${ }^{12}$ Also, Mannich bases of various bioactive compounds have been prepared as prodrugs as a means of overcoming some of their limitations. ${ }^{12}$

Optically-pure chiral Mannich bases of 2-naphthol are particularly popular in metal-mediated and ligand-accelerated catalysis of enantioselective carbon-carbon bond formation. ${ }^{13-19}$ Many of these reactions involve the use of organozinc compounds as alkylating agents which are relatively unreactive if uncoordinated. ${ }^{13}$ These ligands may be used in catalytic amounts. The first synthesis of racemic Mannich-bases of 2-naphthol was achieved by Betti at the turn of the twentieth century. ${ }^{20}$ Thereafter numerous modifications of this reaction surfaced. ${ }^{21-27}$ Since these compounds have multiple centres for chelation with metal ions, they are likely to be potent 
inhibitors of metallo-enzymes. ${ }^{28-31}$ Also, these compounds have the potential to be used as scavengers in cases of heavy metal poisoning. ${ }^{32,33}$

Hence, it is paramount to develop synthetic strategies around the naphthalene nucleus to gain easy access to variety of naphthalene derivatives. Previously, a novel and convenient procedure for the formation of Mannich bases of 2-naphthol under solvent free conditions was reported by our group. ${ }^{26,27}$ As part of the extension of this project, we herein report a microwave-assisted convenient and expeditious method for synthesizing $N, N^{\prime}$-bis-[aryl-(2-hydroxynaphthalen-1-yl)methyl]piperazines (1) and $N, N^{\prime}$-bis(arylmethyl)- $N, N^{\prime}$-bis(2-hydroxynaphthalen-1-yl-methyl)ethylenediamines (2).

\section{Results and Discussion}

Reactions between 2-naphthol, aromatic aldehydes and piperazine resulted in the synthesis of $N, N$ '-bis-[aryl-(2-hydroxynaphthalen-1-yl)-methyl]piperazines (Scheme 1). These reactions were studied under two conditions, as follows: A) Solvent-free microwave irradiation using CEM Discover S Class microwave oven at $125^{\circ} \mathrm{C}$ for five minutes in absence of any catalyst; B) Reflux in ethanol for $72 \mathrm{~h}$ in presence of catalytic amount of pTSA. Two $N, N$ '-bis(arylmethyl)ethylenediamines were also prepared utilizing non-cyclic secondary diamines in place of piperazine under microwave-assisted reactions (Scheme 2).

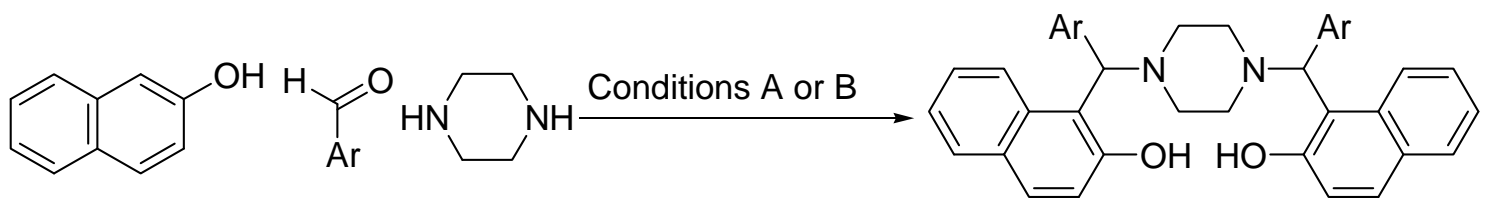

1a-I

Scheme 1. Synthesis of $N, N^{\prime}$-bis[aryl-(2-hydroxynaphthalen-1-yl)-methyl]-piperazines (1a-l). Conditions are depicted in Table 1.

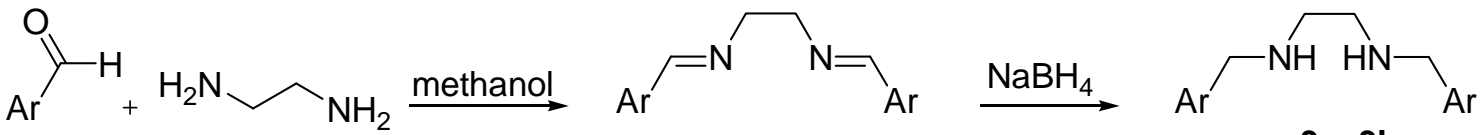

$3 a, 3 b$

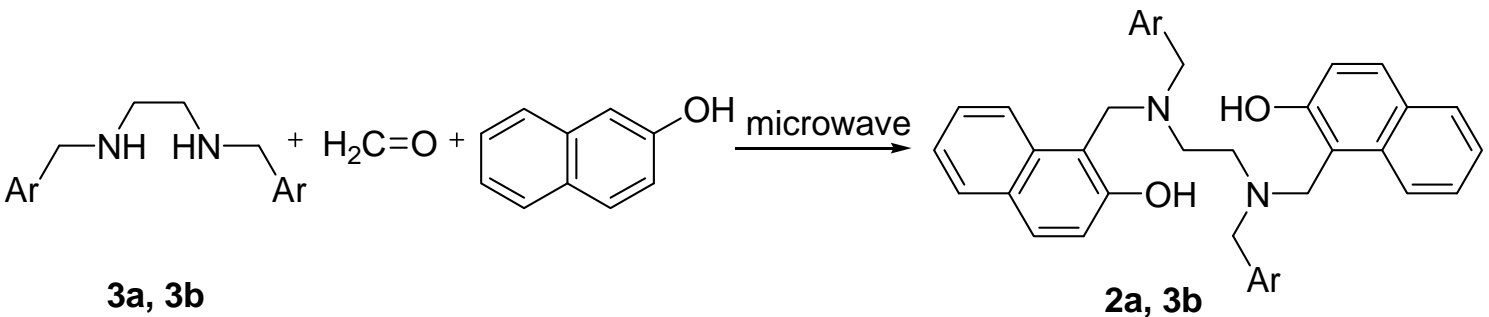

Scheme 2. Synthesis of $N, N^{\prime}$-bis(arylmethyl)- $N, N^{\prime}$-bis(2-hydroxynaphthalen-1-yl-methyl)ethylenediamines (2a,b). 
Table 1. Physical data of $N, N^{\prime}$-bis-[aryl-(2-hydroxynaphthalen-1-yl)-methyl]-piperazines (1a-l) and $N, N^{\prime}$-bis(arylmethyl)- $N, N^{\prime}$-bis(2-hydroxynaphthalen-1-yl-methyl)-ethylenediamines (3a-b)

\begin{tabular}{|c|c|c|c|c|c|}
\hline \multirow{3}{*}{ Product } & \multirow{3}{*}{$\mathrm{Ar}$} & \multicolumn{4}{|c|}{ Reaction Method } \\
\hline & & \multicolumn{2}{|c|}{ Microwave [A] } & \multicolumn{2}{|c|}{ Reflux [B] } \\
\hline & & M.p $\left({ }^{\circ} \mathrm{C}\right)$ & $\%$ Yield & M.p $\left({ }^{\circ} \mathrm{C}\right)$ & $\%$ Yield \\
\hline $1 \mathbf{a}$ & & $241-242$ & 87 & $247-248$ & $81,60^{\mathrm{a}}$ \\
\hline $1 b$ & & $234-235$ & 90 & $240-241$ & 73 \\
\hline 1c & & $243-244$ & 84 & $243-244$ & 51 \\
\hline 1d & & $238-239$ & 93 & $249-250$ & 74 \\
\hline 1e & & $248-250$ & 81 & $254-255$ & 50 \\
\hline 1f & & 214-215 & 81 & $219-222$ & 76 \\
\hline $1 g$ & & $225-226$ & 40 & $243-245$ & 65 \\
\hline $1 \mathrm{~h}$ & & $231-232$ & 95 & $239-240$ & 31 \\
\hline $\mathbf{1 i}$ & & $229-230$ & 56 & $237-238$ & 65 \\
\hline $\mathbf{1 j}$ & & $240-241$ & 93 & $255-256$ & 53 \\
\hline $1 \mathbf{k}$ & & $215-216$ & 92 & $219-220$ & 51 \\
\hline 11 & & $211-213$ & 83 & $213-214$ & 58 \\
\hline 3a & & $152-154$ & 71 & - & - \\
\hline $3 \mathbf{b}$ & & $162-163$ & 74 & - & - \\
\hline
\end{tabular}

${ }^{a}$ Product yield from reaction without catalyst ( $p$ TSA).

All final compounds reported in this paper are new to the chemical literature and were completely characterized by spectroscopic means. To determine the actual spatial arrangement of atoms in the 3-D space, $\mathrm{x}$-ray crystallography of compound $\mathbf{3 b}$ was performed (Figure 1). The resolved structure shows the molecule in its most relaxed form with 2-naphthol and tolyl rings projecting in opposite directions. The H-bonding between phenolic $\mathrm{H}$ and $\mathrm{N}$ is clearly indicated at the two sites. 


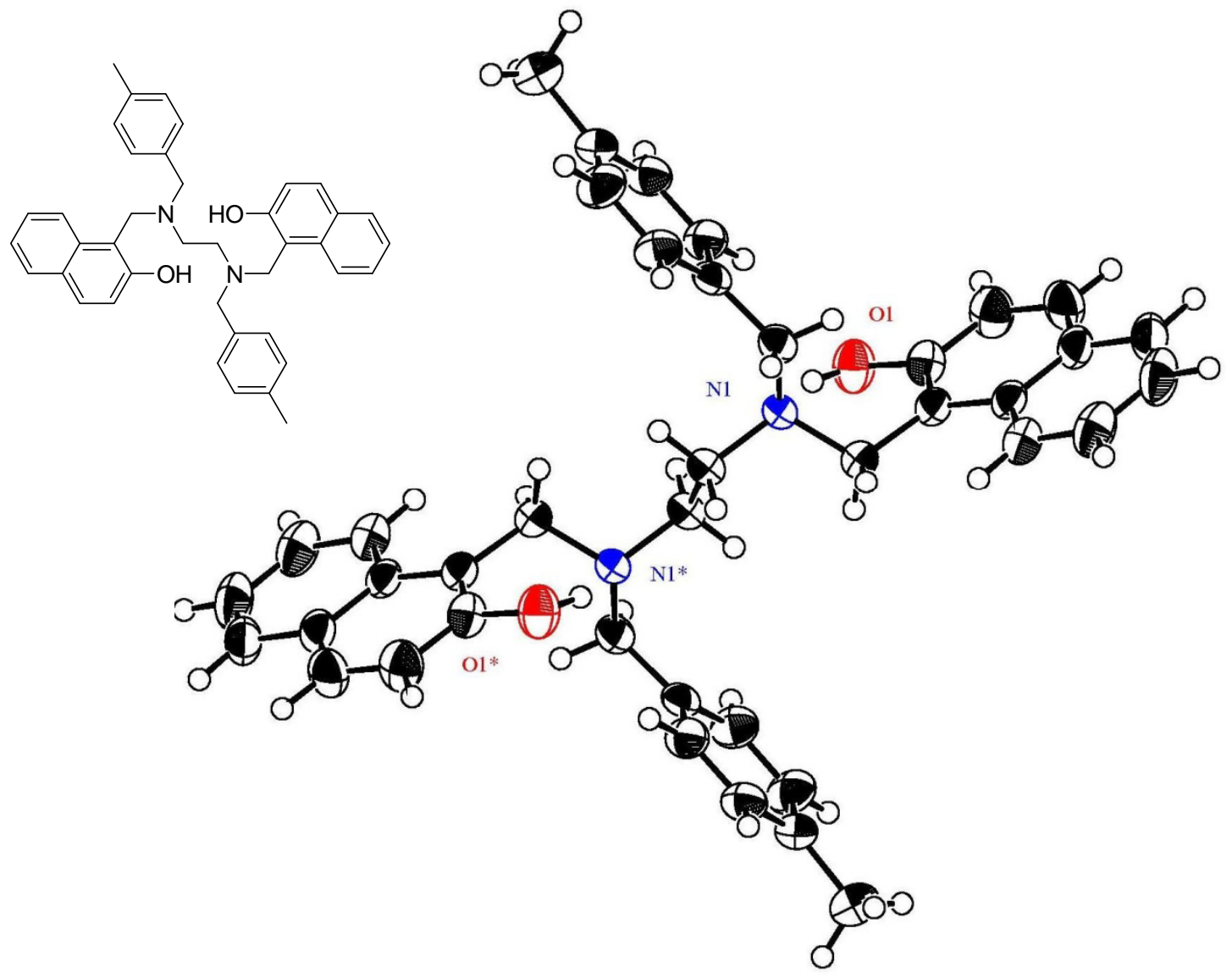

Figure 1. The ORTEP diagram of compound $\mathbf{3 b}$ as obtained by X-ray crystallography.

The popularity of employing microwave energy in organic synthesis has tremendously increased in past decade owing to the simplicity, rapidity, high turnover and green nature of the reactions. ${ }^{34-36}$ Our previous endeavour which led to formation of simple Mannich bases of 2naphthol under solvent-free conditions involved the use of a conventional kitchen microwave and $p$ TSA as catalyst. ${ }^{26,27}$ As evident from data presented in Table 1 , we were able to obtain bisMannich bases 1a-l in good to excellent yields in absence of any catalyst using neat conditions under microwave irradiation; conventional reflux reactions in ethanol benefitted from the use of pTSA (Table 1, entry 1a). The comparison of isolated yields, reaction time and material requirements of the two conditions employed showed microwave-assisted solvent-free reactions as the most efficient synthetic method in terms of energy and time consumption. It should be noted that none of the conditions herein presented were optimized, and the products obtained were not subjected to extensive purification. The products obtained through the reflux reaction protocol had the inherent advantage of digestion of insoluble product precipitates and therefore the purity of the obtained product were consistently better as evidenced by the sharper and higher melting point as compared to same products obtained by employing other conditions. 
The final products in Scheme 1 (1a-l) contained two chiral centers meaning that three diastereomeric forms were possible. More specifically, the final product may include a pair of enantiomers and a meso stereoisomer. This was clearly observed in ${ }^{1} \mathrm{H}$ NMR spectra of a number of analogs where the $\mathrm{H}$ at the stereogenic centre appeared as two discrete peaks (Figure 3; speactra at 300 and $323 \mathrm{~K}$ ). In addition, we observed some difficulty with solubility for all compounds while trying to obtain NMR spectra. Interestingly, the isolated products were fairly soluble in $\mathrm{CDCl}_{3}$ but precipitated after prolonged standing $(\sim 5 \mathrm{~h})$. The precipitated compounds had poor solubility in $\mathrm{CDCl}_{3}$ although the NMR spectra of dilute sample remained unchanged. This led us to speculate that the compounds were crystallizing in a new crystal lattice, exhibiting polymorphism. The precipitation of the compounds after prolonged standing also caused difficulty in recording ${ }^{13} \mathrm{C}$ NMR spectra where molecular dynamics ${ }^{27}$ was already causing disappearance of some peaks at room temperature (vide infra); the problem was compounded by frequent appearance of two peaks for some of the carbons since the products were diastereomeric mixtures.

In ${ }^{1} \mathrm{H}-\mathrm{NMR}$, all compounds clearly showed strong hydrogen-bonding between phenolic $\mathrm{H}$ and neighboring N. However, peak broadening was causing ambiguity in the peak assignment. Both ${ }^{1} \mathrm{H}$ and ${ }^{13} \mathrm{C}$ NMR spectra of compounds suffered from peak broadening owing to molecular dynamics characteristic of cyclic amines. ${ }^{27}$ To improve the resolution of the NMR spectra, variable temperature NMR experiments were conducted on compound 1b (Figures 2-4). Recording of NMR spectra at lower temperature (253K) did improve the resolution but the peaks were still relatively broad.

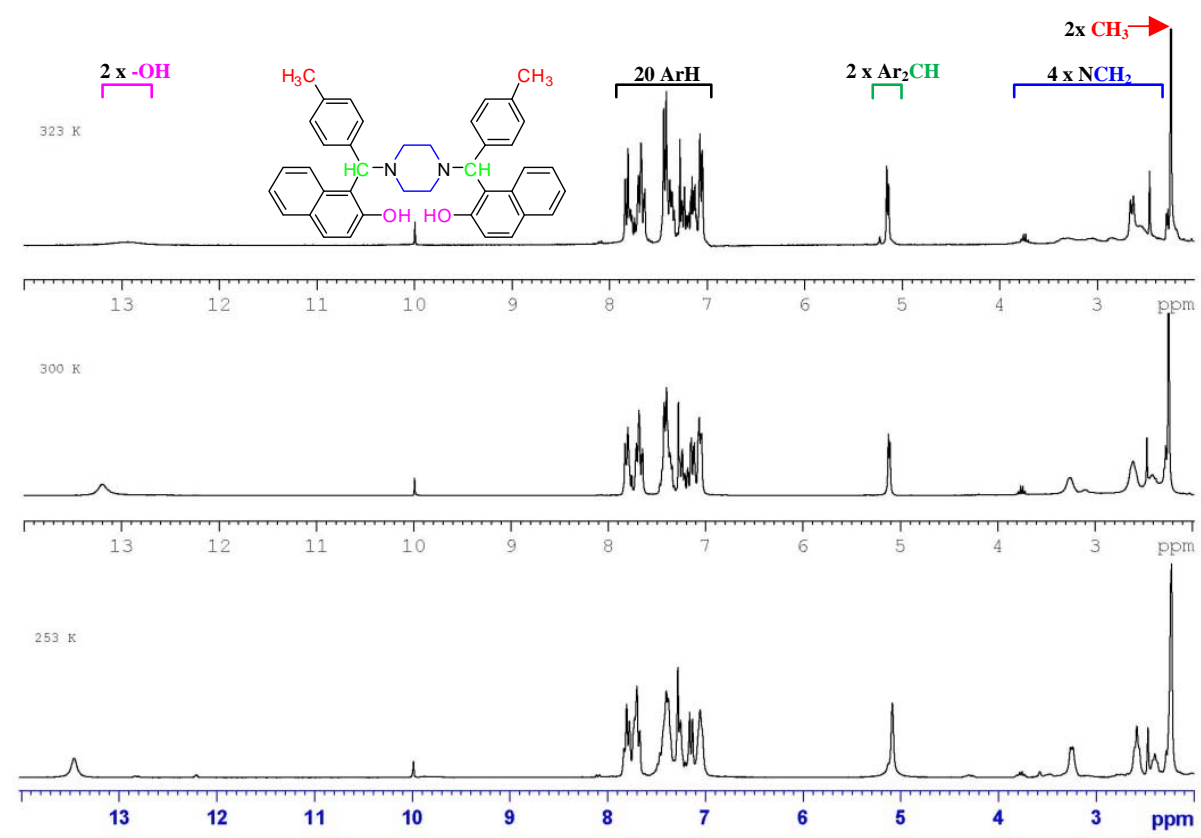

Figure 2. Variable temperature ${ }^{1} \mathrm{H}-\mathrm{NMR}$ stack plots of compound $\mathbf{1 b}$. 


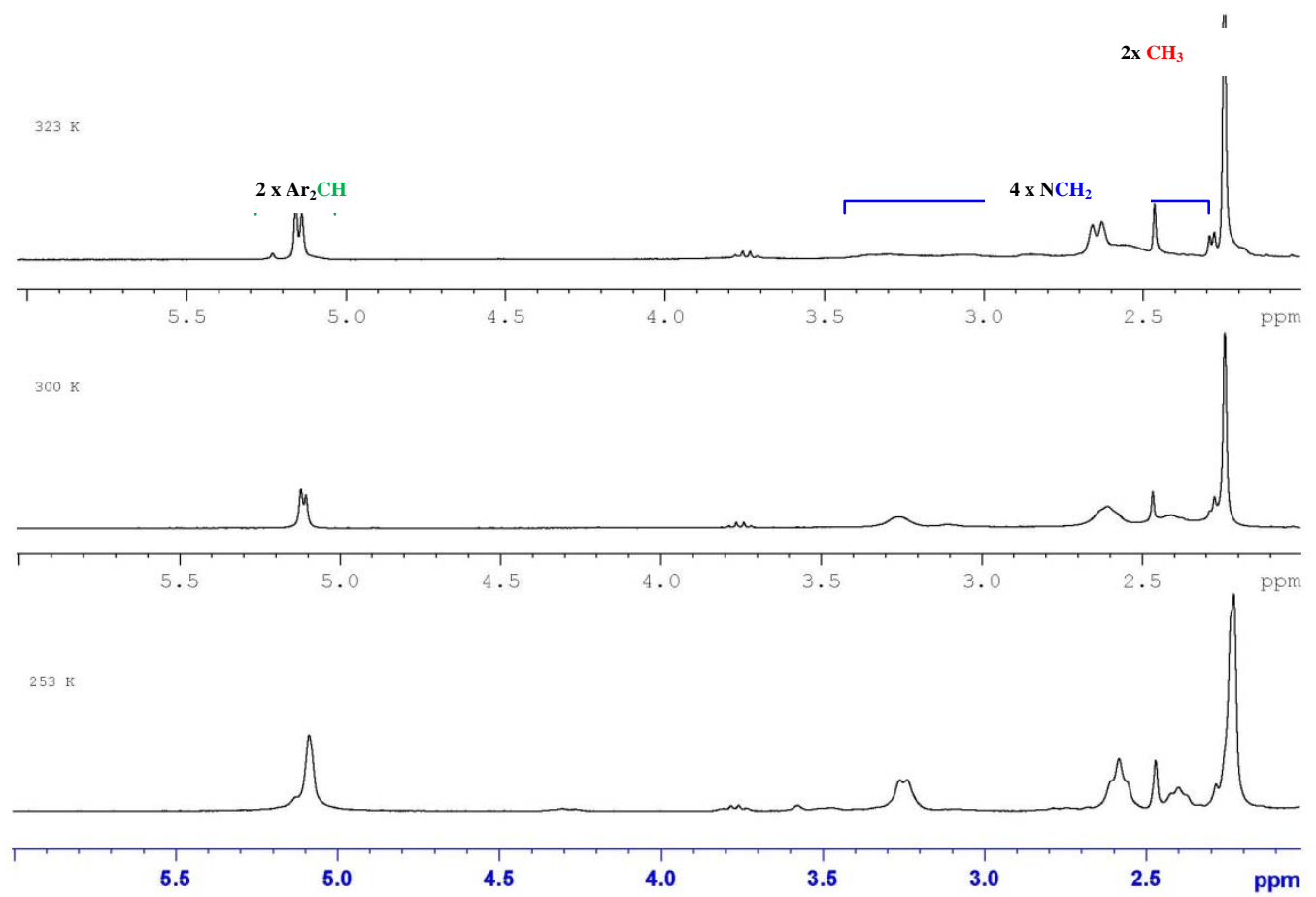

Figure 3. Variable temperature ${ }^{1} \mathrm{H}-\mathrm{NMR}$ stack-plot of aliphatic region of $\mathbf{1 b}$.
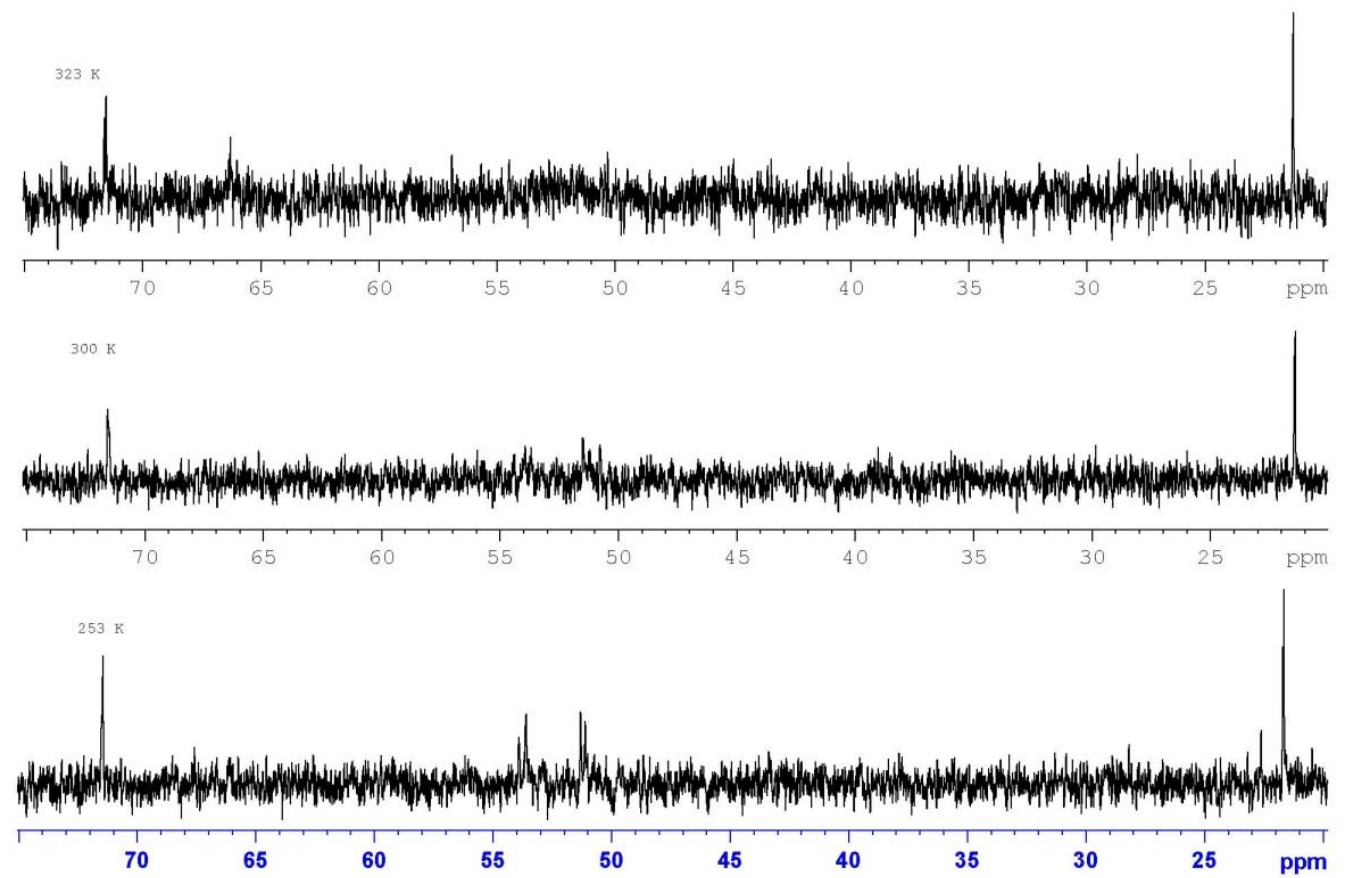

Figure 4. Variable temperature ${ }^{13} \mathrm{C}-\mathrm{NMR}$ stack-plot of aliphatic region of $\mathbf{1 b}$. 
From the Figure 2, the H-bonds (12.8-13.6 ppm) were shown to be weakened by an increase in temperature. The protons and carbons on the piperzine ring displayed molecular dynamics presumably because of the possibility of restricted $\mathrm{N}$-inversion at the two centers. The diastereotopic protons of piperazine appeared as broad peaks in the range 2.3-3.3 ppm at room temperature $(\sim 300 \mathrm{~K})$ and above. These peaks were better resolved at lower temperatures (Figure $3)$. Interestingly with temperature increase, the 2 benzylic protons of enantiomeric pair and the meso diastereomer appeared to resolve better (5.0-5.2 ppm; Figure 3). The carbons on the piperazine ring did not display on ${ }^{13} \mathrm{C}$ NMR spectrum which was recorded at $300 \mathrm{~K}$ or above (presumably because of peak broadening). At lower temperatures, not only did the ${ }^{13} \mathrm{C} \mathrm{NMR}$ spectrum display the well resolved peaks, but also it showed discrete peaks in the range 50-55 ppm for each of the four carbons on the heterocyclic rings (Figure 4). This implied the magnetic non-equivalence of these carbons at experimental temperature due to distortion and loss of symmetry of the molecule.

In conclusion, fourteen bis-Mannich bases of 2-naphthol were successfully synthesized and purified. Although reflux conditions provided products with higher purity, the use of microwaveassisted conditions was shown to be the most efficient method of synthesizing compounds of this type in terms of atom economy, energy consumption and time required. Compounds displayed interesting molecular dynamic as evident by variable temperature NMR and peaks were found to be better resolved at sub-zero temperatures. These compounds may find use as catalysts or pharmaceuticals owing to their potential for metal chelation.

\section{Experimental Section}

General Procedures. All chemicals were obtained from Aldrich Chemical Co. Column chromatographic purifications were undertaken using silica gel (230-400 mesh) obtained from Silicycle. ${ }^{1} \mathrm{H}$ and ${ }^{13} \mathrm{C}$ NMR were recorded on Bruker AV500 and AV300 NMR spectrometers. ESI-HRMS spectra were obtained on Bruker microTOF instrument with an ESI source. Melting points were recorded on an electro-thermal apparatus and are uncorrected. UV-Vis and IR spectra were recorded on LKB Biochrom Ultraspec Plus 4054 and Nicolet Avatar 330 FT-IR spectrophotometers respectively. CEM Discover S-Class microwave reactor was used for the microwave-assisted reactions.

\section{General synthesis of $\boldsymbol{N}, \boldsymbol{N}^{\prime}$-Bis-[aryl-(2-hydroxynaphthalen-1-yl)-methyl]-piperazines (1a-l)}

2-Naphthol $(0.01 \mathrm{~mol})$, piperazine $(0.005 \mathrm{~mol})$, and appropriate aromatic aldehydes $(0.01 \mathrm{~mol})$ were combined and two reaction conditions were employed.

A. Solvent-free microwave irradiation using CEM Discover S Class microwave oven at $125^{\circ} \mathrm{C}$ for five minutes in absence of any catalyst.

B. In the reflux conditions, reaction mixtures in ethanol $(40 \mathrm{ml})$ were refluxed for 72 hours. Catalytic amount of pTSA was added. 
After the reaction proceeded for a stated period of time, respective of the reaction conditions, the insoluble products were sonicated in cold ethanol, filtered and air dried. Appearance, yield and melting points of products obtained under condition B are reported with experimental data.

$\boldsymbol{N}, \boldsymbol{N}$ '-Bis-[(2-hydroxynaphthalen-1-yl)-phenyl-methyl]piperazine (1a). White powder; yield: 81\%; m.p: 247-248 ${ }^{\circ} \mathrm{C} .{ }^{1} \mathrm{H}$ NMR (300 MHz; $\left.\mathrm{CDCl}_{3}\right): \delta 2.44-2.60$ (m, 6H, 6x-NCH), 3.28 (bs, 2H, 2x-NCH), 5.15 (s, 2H, 2x-NCHAr 2 ), 7.12-7.27 (m, 5H, Ar-H), 7.39 (t, J=7.7Hz, 1H, Ar-H), 7.55 (d, J=7.5Hz, 2H, Ar-H), 7.69 (t, J=9.1Hz, 2H, Ar-H), 7.83 (d, J=8.6Hz, 2H, Ar-H), 13.20 (s, $2 \mathrm{H}, 2 \mathrm{x}-\mathrm{OH})$. IR (KBr; $\left.v_{\max }\right): 3416,2120,1635,1270,1119,745 \mathrm{~cm}^{-1}$. UV (EtOH, $\left.\lambda_{\max }\right): 232$, $282 \mathrm{~nm}$. ESI HRMS (amu): measured for $\mathrm{C}_{38} \mathrm{H}_{34} \mathrm{~N}_{2} \mathrm{O}_{2}[\mathrm{M}+\mathrm{H}]^{+}$551.2693; actual [M+H] 551.2699 .

$\boldsymbol{N}, \boldsymbol{N}^{\prime}$-Bis-[(2-hydroxynaphthalen-1-yl)-(4-toluyl)-methyl]piperazine (1b). White powder; yield: 73\%; m.p: 240-241 ${ }^{\circ} \mathrm{C} .{ }^{1} \mathrm{H}$ NMR $\left(300 \mathrm{MHz} ; \mathrm{CDCl}_{3}\right): \delta 2.24$ (s, 6H, $\left.\mathrm{CH}_{3}\right), 2.46-2.61(\mathrm{~m}$, 6H, 6x-NCH), 3.25 (s, 2H, 2x-NCH), 5.11 \& 5.12 (s, 1H each, 2x-NCHAr 2 ), 7.04-7.24 (m, 12H, Ar-H), 7.35-7.45 (m, 4H, Ar-H), 7.64-7.82 (m, 4H, Ar-H), 13.20(s, 2H, 2x-OH). IR (KBr; $\left.v_{\max }\right)$ : 3418, 2099, 1636, $667 \mathrm{~cm}^{-1}$. UV (EtOH, $\left.\lambda_{\max }\right)$ : 227, $280 \mathrm{~nm}$. ESI HRMS (amu): measured for $\mathrm{C}_{40} \mathrm{H}_{38} \mathrm{~N}_{2} \mathrm{O}_{2}[\mathrm{M}+\mathrm{H}]^{+}$579.3006; actual $[\mathrm{M}+\mathrm{H}]^{+} 579.3012$.

$N, N$ '-Bis-[(4-fluorophenyl)-(2-hydroxynaphthalen-1-yl)-methyl]piperazine $\quad$ (1c). Beige powder; yield: 51\%; m.p: 243-244 ${ }^{\circ} \mathrm{C} .{ }^{1} \mathrm{H}$ NMR (300 MHz; $\mathrm{CDCl}_{3}$ ): $\delta$ 2.32-2.60 (m, 6H, 6x$\mathrm{NCH}), 3.28(\mathrm{~s}, 2 \mathrm{H}, 2 \mathrm{x}-\mathrm{NCH}), 5.14$ \& 5.15 (s, 1H each, 2x-NCHAr $), 6.96(\mathrm{t}, \mathrm{J}=8.4 \mathrm{~Hz}, 4 \mathrm{H}, \mathrm{Ar}-$ H), 7.11-7.16 (m, 2H, Ar-H), 7.24-7.29 (m, 2H, Ar-H), 7.38-7.43 (m, 2H, Ar-H), 7.50-7.55 (m, 2H, Ar-H), 7.67-7.79 (m, 8H, Ar-H), 13.10 (s, 2H, 2x-OH). IR (KBr; v $\max$ ): 3414, 2099, 1634, $667 \mathrm{~cm}^{-1}$. UV (EtOH, $\left.\lambda_{\max }\right): 232,280 \mathrm{~nm}$. ESI HRMS (amu): measured for $\mathrm{C}_{38} \mathrm{H}_{32} \mathrm{~F}_{2} \mathrm{~N}_{2} \mathrm{O}_{2}$ $[\mathrm{M}+\mathrm{H}]^{+}$587.2505; actual $[\mathrm{M}+\mathrm{H}]^{+}$587.2510.

$\boldsymbol{N}, \boldsymbol{N}$ '-Bis-[(4-chlorophenyl)-(2-hydroxynaphthalen-1-yl)-methyl]piperazine (1d). White powder; yield: 74\%; m.p: 249-250 ${ }^{\circ} \mathrm{C} .{ }^{1} \mathrm{H}$ NMR (300 MHz; $\left.\mathrm{CDCl}_{3}\right): \delta$ 2.29-2.61 (m, 6H, 6x$\mathrm{NCH}$ ), 3.27 (s, 2H, 2x-NCH), 5.13 \& 5.14 (s, 1H each, 2x-NCHAr 2 ), 7.11-7.15 (m, 2H, Ar-H), 7.24 (d, J=8.1Hz, 2H, Ar-H), 7.41 (t, J=7.5Hz, 2H, Ar-H), 7.49 (d, J=8.4Hz, 4H, Ar-H), 7.677.86 (m, 8H, Ar-H), 12.90 (s, 2H, 2x-OH). IR (KBr; $\left.v_{\max }\right): 3422,2108,1634,1409,1225,947$ $\mathrm{cm}^{-1}$. UV (EtOH, $\left.\lambda_{\max }\right): 227 \mathrm{~nm}$. ESI HRMS (amu): measured for $\mathrm{C}_{38} \mathrm{H}_{32} \mathrm{Cl}_{2} \mathrm{~N}_{2} \mathrm{O}_{2}[\mathrm{M}+\mathrm{H}]^{+}$ 619.1914; actual $[\mathrm{M}+\mathrm{H}]^{+} 619.1919$.

$N, N$ '-Bis-[(2-hydroxynaphthalen-1-yl)-(4-methoxyphenyl)-methyl]piperazine

(1e). Yellowish orange powder; yield: 50\%; m.p: $254-255{ }^{\circ} \mathrm{C} .{ }^{1} \mathrm{H}$ NMR $\left(300 \mathrm{MHz} ; \mathrm{CDCl}_{3}\right): \delta 2.29$ 2.61(m, 6H, 6x-NCH), $3.25(\mathrm{~s}, 2 \mathrm{H}, 2 \mathrm{x}-\mathrm{NCH}), 3.72$ (s, 6H, 2x-OCH $), 5.10\left(\mathrm{~s}, 2 \mathrm{H}, 2 \mathrm{x}-\mathrm{NCHAr}_{2}\right)$, 6.78 (dd, J=8.7, 2.1 Hz, 4H, Ar-H), 7.11-7.14 (m, 2H, Ar-H), 7.20-7.28 (m, 2H, Ar-H), 7.35-7.46 (m, 6H, Ar-H), 7.65-7.72 (m, 2H, Ar-H), 7.80 (d, J=8.7Hz, 2H, Ar-H), 13.20 (s, 2H, 2x-OH). IR $\left(\mathrm{KBr} ; v_{\max }\right): 3440,2966,2843,2095,1635,1258,1119,947 \mathrm{~cm}^{-1}$. UV (EtOH, $\left.\lambda_{\max }\right): 229,281$ nm. ESI HRMS (amu): measured for $\mathrm{C}_{40} \mathrm{H}_{38} \mathrm{~N}_{2} \mathrm{O}_{4}[\mathrm{M}+\mathrm{H}]^{+}$611.2904; actual [M+H] $]^{+}$611.2910.

$\boldsymbol{N}, \boldsymbol{N}^{\prime}$-Bis-[(2-hydroxynaphthalen-1-yl)-(4-nitrophenyl)-methyl]piperazine $\quad$ (1f). White powder; yield: 76\%; m.p: 219-222 ${ }^{\circ} \mathrm{C} .{ }^{1} \mathrm{H}$ NMR $\left(300 \mathrm{MHz} ; \mathrm{CDCl}_{3}\right): \delta$ 2.51-2.66 (m, 6H, 6x$\mathrm{NCH}), 3.35$ (s, 2H, 2x-NCH), 5.25 \& 5.29 (s, 1H each, 2x-NCHAr 2 ), 7.11-7.16 (m, 2H, Ar-H), 
$7.43(\mathrm{t}, \mathrm{J}=7.2 \mathrm{~Hz}, 2 \mathrm{H}, \mathrm{Ar}-\mathrm{H}), 7.69-7.79$ (m, 12H, Ar-H), 8.08-8.16 (m, 4H, Ar-H), 12.55 (bs, 2H, 2x-OH). IR (KBr; $\left.v_{\max }\right): 3413,2908,2381,2108,1635,1521,1115,746 \mathrm{~cm}^{-1}$. UV (EtOH, $\left.\lambda_{\max }\right)$ : 231, $270 \mathrm{~nm}$. ESI HRMS (amu): measured for $\mathrm{C}_{38} \mathrm{H}_{32} \mathrm{~N}_{4} \mathrm{O}_{6}[\mathrm{M}+\mathrm{H}]^{+}$641.2395; actual [M+H] 641.2400 .

$\boldsymbol{N}, N^{\prime}$-Bis-[(2-hydroxynaphthalen-1-yl)-(4-(N,N-dimethylamino)phenyl)-methyl]piperazine (1g). White powder; yield: 65\%; m.p: 243-245 ${ }^{\circ} \mathrm{C} .{ }^{1} \mathrm{H}$ NMR $\left(300 \mathrm{MHz} ; \mathrm{CDCl}_{3}\right): \delta$ 2.18-2.2.40 $(\mathrm{m}, 6 \mathrm{H}, 6 \mathrm{x}-\mathrm{NCH}), 2.65\left(\mathrm{~s}, 12 \mathrm{H}, 4 \mathrm{x}-\mathrm{NCH}_{3}\right), 2.99-3.23(\mathrm{~m}, 2 \mathrm{H}, 2 \mathrm{x}-\mathrm{NCH}), 5.05$ (s, 2H, 2x$\left.\mathrm{NCHAr}_{2}\right), 6.58$ (d, J=8.1Hz, 2H, Ar-H), 7.10-7.14 (d, J=8.7Hz, 2H, Ar-H), 7.22-7.25 (m, 2H, ArH), 7.35 (d, J=7.8Hz, 6H, Ar-H), 7.63-7.70 (m, 4H, Ar-H), 7.82 (d, J=8.7Hz, 2H, Ar-H), 13.31 (s, 2H, 2x-OH). IR (KBr; $\left.v_{\max }\right): 3425,2115,1639,1520,1454,951,814 \mathrm{~cm}^{-1}$. UV (EtOH, $\lambda_{\max }$ ): 232, $282 \mathrm{~nm}$. ESI HRMS (amu): measured for $\mathrm{C}_{42} \mathrm{H}_{44} \mathrm{~N}_{4} \mathrm{O}_{2}[\mathrm{M}+\mathrm{H}]^{+}$637.3539; actual [M+H] 637.3543.

$N, N^{\prime}$-Bis-[(3,4-dichlorophenyl)-(2-hydroxynaphthalen-1-yl)-methyl]piperazine (1h). White powder; yield: 70\%; m.p: 239-240 ${ }^{\circ} \mathrm{C} .{ }^{1} \mathrm{H}$ NMR (300 MHz; $\left.\mathrm{CDCl}_{3}\right): \delta$ 2.42-2.78 (m, 6H, 6x$\mathrm{NCH}), 3.29$ (s, 2H, 2x-NCH), $5.11 \& 5.12$ (s, 1H each, 2x-NCHAr $), 7.10-7.13(\mathrm{~m}, 2 \mathrm{H}, \mathrm{Ar}-\mathrm{H})$, 7.26-7.46 (m, 8H, Ar-H), 7.64-7.75 (m, 8H, Ar-H), 12.65 \& 12.70 (bs, 1H each, 2x-OH). IR $\left(\mathrm{KBr} ; v_{\max }\right): 3402,2115,1653,668 \mathrm{~cm}^{-1}$. UV (EtOH, $\left.\lambda_{\max }\right): 230,284 \mathrm{~nm}$. ESI HRMS (amu): measured for $\mathrm{C}_{38} \mathrm{H}_{30} \mathrm{Cl}_{4} \mathrm{~N}_{2} \mathrm{O}_{2}[\mathrm{M}+\mathrm{H}]^{+}$687.1134; actual [M+H] $]^{+} 687.1140$.

$\boldsymbol{N}, \boldsymbol{N}^{\prime}$-Bis-[(2-hydroxynaphthalen-1-yl)-(3,4-dimethoxyphenyl)-methyl]piperazine (1i). White powder; yield: 65\%; m.p: 230-231 ${ }^{\circ} \mathrm{C}$. ${ }^{1} \mathrm{H}$ NMR (300 MHz; $\left.\mathrm{CDCl}_{3}\right): \delta 2.50-2.61$ (m, 6H, 6x$\mathrm{NCH}), 3.15$ (s, 2H, 2x-NCH), 3.79 (s, 12H, 4x-OCH 3$), 5.09$ (s, 2H, 2x-NCHAr $), 6.75$ (d, $\mathrm{J}=8.4 \mathrm{~Hz}, 2 \mathrm{H}, \mathrm{Ar}-\mathrm{H}), 7.08-7.15(\mathrm{~m}, 6 \mathrm{H}, \mathrm{Ar}-\mathrm{H}), 7.25-7.29(\mathrm{~m}, 4 \mathrm{H}, \mathrm{Ar}-\mathrm{H}), 7.40$ (t, J=9.0Hz, 2H, Ar-H), 7.66-7.73 (m, 4H, Ar-H), 7.83 (d, J=8.7Hz, 2H, Ar-H), 13.15 (s, 2H, 2x-OH). IR (KBr; $\left.v_{\max }\right): 3404,2112,1642,1266,939 \mathrm{~cm}^{-1}$. UV (EtOH, $\left.\lambda_{\max }\right): 232,282 \mathrm{~nm}$. ESI HRMS (amu): measured for $\mathrm{C}_{42} \mathrm{H}_{42} \mathrm{~N}_{2} \mathrm{O}_{6}[\mathrm{M}+\mathrm{H}]^{+} 671.3116$; actual $[\mathrm{M}+\mathrm{H}]^{+} 671.3121$.

$\boldsymbol{N}, \boldsymbol{N}^{\prime}$-Bis-[(2-hydroxynaphthalen-1-yl)-(3,4-methylenedioxyphenyl)-methyl]piperazine (1j). White powder; yield: 53\%; m.p: 250-251 ${ }^{\circ} \mathrm{C} .{ }^{1} \mathrm{H}$ NMR $\left(300 \mathrm{MHz} ; \mathrm{CDCl}_{3}\right): \delta 2.41-2.66(\mathrm{~m}, 6 \mathrm{H}$, $6 \mathrm{x}-\mathrm{NCH}), 3.24(\mathrm{~s}, 2 \mathrm{H}, 2 \mathrm{x}-\mathrm{NCH}), 5.07 \& 5.09$ (s, 1H each, 2x-NCHAr 2$), 5.84 \& 5.89(\mathrm{~s}, 2 \mathrm{H}$ each, 2x-OCH$\left.{ }_{2} \mathrm{O}-\right), 6.70(\mathrm{~d}, \mathrm{~J}=8.1 \mathrm{~Hz}, 2 \mathrm{H}, \mathrm{Ar}-\mathrm{H}), 6.97-7.16(\mathrm{~m}, 6 \mathrm{H}, \mathrm{Ar}-\mathrm{H}), 7.23-7.26(\mathrm{~m}, 2 \mathrm{H}$, Ar-H), 7.36-7.42 (m, 2H, Ar-H), 7.65-7.73 (m, 4H, Ar-H), 7.80 (d, J=8.7Hz, 2H, Ar-H), 13.09 \& 13.10 (bs, $1 \mathrm{H}$ each, 2x-OH). IR (KBr; $\left.v_{\max }\right): 3557,2109,1634,1241,1029 \mathrm{~cm}^{-1}$. UV (EtOH, $\lambda_{\text {max }}$ ): $229,285 \mathrm{~nm}$. ESI HRMS (amu): measured for $\mathrm{C}_{40} \mathrm{H}_{34} \mathrm{~N}_{2} \mathrm{O}_{6}[\mathrm{M}+\mathrm{H}]^{+}$639.2490; actual $[\mathrm{M}+\mathrm{H}]^{+} 639.2495$.

$\boldsymbol{N}, \boldsymbol{N}$ '-Bis-[(2-hydroxynaphthalen-1-yl)-(4-pyridyl)-methyl]piperazine (1k). White powder; yield: \%; m.p: $219-220{ }^{\circ} \mathrm{C} .{ }^{1} \mathrm{H}$ NMR $\left(300 \mathrm{MHz} ; \mathrm{CDCl}_{3}\right): \delta$ 2.45-2.79 (m, 6H, 6x-NCH), 3.31 (s, 2H, 2x-NCH), 5.48 (s, 2H, 2x-NCHAr $)$ ), 7.10-7.14 (m, 4H, Ar-H), 7.25 (d, J=9.0Hz, 2H, Ar-H), $7.42(\mathrm{t}, \mathrm{J}=6.6 \mathrm{~Hz}, 2 \mathrm{H}, \mathrm{Ar}-\mathrm{H}), 7.56-7.72(\mathrm{~m}, 8 \mathrm{H}, \mathrm{Ar}-\mathrm{H}), 8.05$ (d, J=9.0Hz, 2H, Ar-H), 8.54 (d, $\mathrm{J}=3.9 \mathrm{~Hz}, 2 \mathrm{H}, \mathrm{Ar}-\mathrm{H}), 12.85$ (s, 2H, 2x-OH). IR (KBr; $\left.v_{\max }\right): 3385,2113,1634,1466,951 \mathrm{~cm}^{-1}$. UV (EtOH, $\lambda_{\max }$ ): $229 \mathrm{~nm}$. ESI HRMS (amu): measured for $\mathrm{C}_{36} \mathrm{H}_{32} \mathrm{~N}_{4} \mathrm{O}_{2}[\mathrm{M}+\mathrm{H}]^{+} 553.2598$; actual $[\mathrm{M}+\mathrm{H}]^{+} 553.2604$. 
$\boldsymbol{N}, \boldsymbol{N}^{\prime}$-Bis-[(2-hydroxynaphthalen-1-yl)-(2-pyridyl)-methyl]piperazine (11). Creamy yellow powder; yield: \%; m.p: 210-212 ${ }^{\circ} \mathrm{C} .{ }^{1} \mathrm{H}$ NMR (300 MHz; $\left.\mathrm{CDCl}_{3}\right): \delta 2.45-2.79(\mathrm{~m}, 6 \mathrm{H}, 6 \mathrm{x}-\mathrm{NCH})$, 3.31 (s, 2H, 2x-NCH), 5.15 \& 5,16 (s, 1H each, 2x-NCHAr $)$, 7.10-7.15 (m, 4H, Ar-H), 7.25 (d, $\mathrm{J}=9.0 \mathrm{~Hz}, 2 \mathrm{H}, \mathrm{Ar}-\mathrm{H}), 7.44(\mathrm{t}, \mathrm{J}=9.0 \mathrm{~Hz}, 2 \mathrm{H}, \mathrm{Ar}-\mathrm{H}), 7.51(\mathrm{~d}, \mathrm{~J}=5.7 \mathrm{~Hz}, 4 \mathrm{H}, \mathrm{Ar}-\mathrm{H}), 7.68-7.80$ (m, 6H, Ar-H), 8.52 (d, J=5.4Hz, 2H, Ar-H), 12.44 \& 12.45 (bs, 1H each, 2x-OH). IR (KBr; $v_{\max }$ ): 3440, 2103, 1638, 1225, $943 \mathrm{~cm}^{-1}$. UV (EtOH, $\left.\lambda_{\max }\right): 227,279 \mathrm{~nm}$. ESI HRMS (amu): measured for $\mathrm{C}_{36} \mathrm{H}_{32} \mathrm{~N}_{4} \mathrm{O}_{2}[\mathrm{M}+\mathrm{H}]^{+}$553.2598; actual $[\mathrm{M}+\mathrm{H}]^{+}$553.2604.

Synthesis of $N, N^{\prime}$-bis(arylmethyl)- $N, N^{\prime}$-bis(2-hydroxynaphthalen-1-yl-methyl)-ethylenediamines (3a,b)

Synthesis of $\boldsymbol{N}, \boldsymbol{N}$ '-bis(arylethane)-1,2-diamine. Reported procedure ${ }^{37}$ was followed for the sythesis of N,N'-bis(arylmethyl)-1,2-ethylenediamine. The diamine $(1.00 \mathrm{mmol})$ and the corresponding aldehyde $(2.00 \mathrm{mmol})$ were dissolved in methanol $(10 \mathrm{~mL})$ and stirred for 1 hour at room temperature and then cooled at $5{ }^{\circ} \mathrm{C}$ in an icebath. The precipitate (imine) was collected and washed extensively with cold methanol. The precipitate was then suspended in ethanol (100 $\mathrm{ml}$ ) follow by addition of 0.67 molar equivalent of $\mathrm{NaBH}_{4}$ portionwise. The reaction mixture was stirred at $55-60^{\circ} \mathrm{C}$ for 2 hour and then allowed to stand at room temperature for an hour. Solvent was rotary evaporated at this point and the crude product was extracted from EtOAc and water mixture. Dried EtOAc layer was rotary evaporated to dryness to produce the product 2.

$\boldsymbol{N}, \boldsymbol{N}$ '-Dibenzylethane-1,2-diamine (2a). Yellow liquid. ${ }^{1} \mathrm{H} \mathrm{NMR}\left(300 \mathrm{MHz} ; \mathrm{CDCl}_{3}\right): \delta 1.59$ (s, $2 \mathrm{H}, \mathrm{NH}), 2.82$ (s, 4H, 2x-NCH 2 ), 3.82 (s, 4H, 2xArCH $2^{-}$), 7.15-7.30 (m, 10H, Ar-H).

$N, N^{\prime}$-Bis(4-methylbenzyl)ethane-1,2-diamine (2b). White powder; m.p. $112-114^{\circ} \mathrm{C}$; lit m.p. $116^{\circ} \mathrm{C} .{ }^{38} \mathrm{H}$ NMR $\left(300 \mathrm{MHz} ; \mathrm{CDCl}_{3}\right): \delta 1.59(\mathrm{~s}, 2 \mathrm{H}, \mathrm{NH}), 2.40\left(\mathrm{~s}, 6 \mathrm{H}, 2 \mathrm{x}-\mathrm{ArCH}_{3}\right), 2.81(\mathrm{~s}, 4 \mathrm{H}$, 2x- $\mathrm{NCH}_{2}$ ), 3.80(s, 4H, 2xArCH $\left.2_{2}\right), 7.19$ (d, J=7.8Hz, 4H, Ar-H), 7.27(d, J=8.1 Hz, 4H, Ar-H).

\section{Synthesis of $N, N^{\prime}$-bis(arylmethyl)- $N, N^{\prime}$-bis(2-hydroxynaphthalen-1-yl-methyl)-ethylene- diamines (3a,b)}

2-Naphthol (1.88 g, $20 \mathrm{mmol}$ ), paraformaldehyde (600 mg, $20 \mathrm{mmol})$, and corresponding N,N'bis(arylmethyl)-1,2-ethylenediamine $(10 \mathrm{mmol})$ were mixed and reacted under solvent-free conventional microwave-assisted conditions at highest power $(900 \mathrm{~W})$ for 2 minutes. Precipitation occurred when ethanol $(15 \mathrm{ml})$ was added. The products were sonicated in cold ethanol, filtered and air dried.

$\boldsymbol{N}, \boldsymbol{N}^{\prime}$-Bis(2-hydroxynaphthalen-1-yl-methyl)- $\boldsymbol{N}, \boldsymbol{N}$ '-bis(phenylmethyl)-ethylenediamine (3a). White powder; yield: 71\%; m.p: $152-154{ }^{\circ} \mathrm{C} .{ }^{1} \mathrm{H}$ NMR $\left(300 \mathrm{MHz} ; \mathrm{CDCl}_{3}\right): \delta 2.87$ (s, 4H, 2x$\mathrm{NCH}_{2} \mathrm{CH}_{2} \mathrm{~N}-$ ), 3.62 (s, 4H, 2x-NCH $2 \mathrm{Ph}$ ), 4.13 (s, 4H, 2x- $\left.\mathrm{NCH}_{2} \mathrm{Naphthyl}\right), 7.10$ (d, J=8.7Hz, 4H, Ar-H), 7.18-7.33 (m, 2H, Ar-H), 7.41 (t, J=6.9Hz, 4H, Ar-H), 7.67-7.78 (m, 12H, Ar-H), 11.95 $(\mathrm{s}, 2 \mathrm{H}, 2 \mathrm{x}-\mathrm{OH}) .{ }^{13} \mathrm{C} \mathrm{NMR}\left(75 \mathrm{MHz} ; 300 \mathrm{~K} ; \mathrm{CDCl}_{3}\right): \delta 50.93,53.42,59.17,111.69,119.41$, 121.32, 122.94, 126.81, 128.24, 129.08, 129.33, 129.81, 129.99, 129.99, 132.98, 136.34, 156.62. IR (KBr; $\left.v_{\max }\right): 3419.2120,1622,814,740 \mathrm{~cm}^{-1}$. UV (EtOH, $\left.\lambda_{\max }\right): 229,277 \mathrm{~nm}$. ESI HRMS (amu): measured for $\mathrm{C}_{38} \mathrm{H}_{36} \mathrm{~N}_{2} \mathrm{O}_{2}[\mathrm{M}+\mathrm{H}]^{+}$553.2850; actual $[\mathrm{M}+\mathrm{H}]^{+}$553.2855. 
$\boldsymbol{N}, \boldsymbol{N}$ '-Bis(2-hydroxynaphthalen-1-yl-methyl)- $N, N^{\prime}$-bis(4-tolylmethyl)ethylenediamine (3b). White powder; yield: 74\%; m.p: $162-163{ }^{\circ} \mathrm{C} .{ }^{1} \mathrm{H}$ NMR $\left(300 \mathrm{MHz} ; \mathrm{CDCl}_{3}\right): \delta 2.31$ (s, $6 \mathrm{H}$, $\left.2 \mathrm{ArCH}_{3}\right), 2.86\left(\mathrm{~s}, 4 \mathrm{H},-\mathrm{NCH}_{2} \mathrm{CH}_{2} \mathrm{~N}-\right), 3.58$ (s, 4H, 2x- $\mathrm{NCH}_{2}$ Toluyl), 4.11 (s, 4H, 2x-

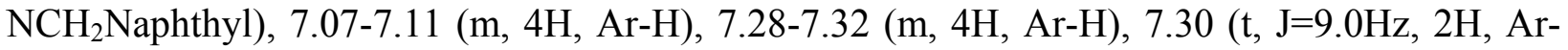
$\mathrm{H}), 7.66-7.77(\mathrm{~m}, 10 \mathrm{H}, \mathrm{Ar}-\mathrm{H}), 11.95(\mathrm{~s}, 2 \mathrm{H}, 2 \mathrm{x}-\mathrm{OH}) .{ }^{13} \mathrm{C} \mathrm{NMR}\left(75 \mathrm{MHz} ; 300 \mathrm{~K} ; \mathrm{CDCl}_{3}\right): \delta$ 21.51, 50.83, 53.31, 58.71, 111.74, 119.44, 121.33, 122.89, 126.76, 128.98, 129.31, 129.31, 129.73, 129.97, 132.98, 133.18, 137.91, 156.68. IR (KBr; $\left.v_{\max }\right): 3409,3051,2132,1623,1266$, $738 \mathrm{~cm}^{-1}$. UV (EtOH, $\left.\lambda_{\max }\right): 229,276 \mathrm{~nm}$. ESI HRMS (amu): measured for $\mathrm{C}_{40} \mathrm{H}_{40} \mathrm{~N}_{2} \mathrm{O}_{2}[\mathrm{M}+\mathrm{H}]^{+}$ 581.3163. actual $[\mathrm{M}+\mathrm{H}]^{+} 581.3168$.

X-Ray crystallography. The purified compound (3b,100 mg) as powder was dissolved in $\mathrm{CHCl}_{3}(10 \mathrm{~mL})$ and allowed to stand for 48 hours. The colourless crystals obtained were washed with cold acetonitrile and air dried. A colorless plate crystal having approximate dimensions of $0.32 \times 0.26 \times 0.18 \mathrm{~mm}$ was mounted on a glass fiber. All measurements were made on a Rigaku Mercury2 CCD area detector with graphite monochromated Mo-K $\alpha$ radiation. Indexing was performed from 6 images that were exposed for 5.0 seconds. The crystal-to-detector distance was $50.10 \mathrm{~mm}$. Cell constants and an orientation matrix for data collection corresponded to a Ccentered monoclinic cell with dimensions: $a=20.9975(8), b=9.3696(4), c=18.4218(7) \AA$, $\beta=117.8154(11)^{\circ}, V=3205.5(2) \AA^{3}$. For $Z=4$ and $F W=580.77$, the calculated density is $1.203 \mathrm{~g} / \mathrm{cm}^{3}$. Based on the systematic absences of $\mathrm{hkl}: \mathrm{h}+\mathrm{k} \pm 2 \mathrm{n}$ and $\mathrm{h} 0 \mathrm{l}: \mathrm{l} \pm 2 \mathrm{n}$ packing considerations, a statistical analysis of intensity distribution, and the successful solution and refinement of the structure, the space group was determined to be $\mathrm{C} 2 / \mathrm{c}$ (\#15).

The data were collected at a temperature of $-50 \pm 1{ }^{\circ} \mathrm{C}$ to a maximum $2 \theta$ value of $55.0^{\circ}$. Of the 16329 reflections that were collected, 3665 were unique $\left(\mathrm{R}_{\text {int }}=0.032\right)$; equivalent reflections were merged. Data were collected and processed using CrystalClear (Rigaku). The structure was solved by direct methods and expanded using Fourier techniques. The non-hydrogen atoms were refined anisotropically. Hydrogen atoms were refined using the riding model. The final cycle of full-matrix least-squares refinement on $\mathrm{F}$ was based on 2256 observed reflections (I>3.00\%(I)) and 220 variable parameters and converged (largest parameter shift was 0.00 times its esd) with unweighted and weighted agreement factors of $\mathrm{R}=0.0363$ and $\mathrm{R}_{\mathrm{W}}=0.0440$. CCDC 700814 contains the supplementary crystallographic data for this paper. These data can be obtained free of charge from The Cambridge Crystallographic Data Centre via www.ccdc.cam.ac.uk/data_request/cif.

\section{Acknowledgements}

We are grateful to Natural Sciences and Engineering Research Council (NSERC) for financial support. Ms C Nichols is thanked for preliminary investigations. The Atlantic Region Magnetic 
Resonance Centre (ARMRC) and Maritime Mass Spectrometry Laboratory (MMSL) Dalhousie University are acknowledged for providing NMR and MS support, respectively.

\section{References}

1. Karll, R. E.; Lee, R. J. US Patent, US 4384 138, 1983.

2. Otto, F. P. US Patent, US 3649 229, 1972.

3. Horodysky, A. G.; Kaminski, J. M. US Patent, US 4394 278, 1983.

4. Lee, L. M; Plattner, J. J.; Ours, C. W.; Horrom, B. W.; Smital, J. R; Martin, Y. C.; Pernet, A. G.; Bunnell, P. R.; El Masry, S. E.; Dodge, P. W. J. Med. Chem. 1984, 27, 1579.

5. Scott, M. K.; Martin, G. E.; DiStefano, D. L.; Fedde, C. L.; Kukla, M. J.; Barrett, D. L.; Baldy, W. J.; Elgin R. J. Jr.; Kesslick, J. M.; Mathiasen, J. R.; Strank, R. P.; Vaught, J. L. J. Med. Chem. 1992, 35, 552.

6. Cohen, A.; Hall, R. A.; Heath-Brown, B.; Parkes, M. W.; Rees, A. H. British J. Pharmacol. Chemother. 1957, 12, 194.

7. Borenstein, M. R.; Doukas, P. H. J. Pharm. Sci. 1987, 76, 300.

8. Shiozawa, A.; Narita, K.; Izumi, G.; Kurashige, S.; Sakitama, K.; Ishikawa, M. Eur. J. Med. Chem. 1995, 30, 85.

9. Barlin, G. B.; Jiravinya, C. Aus. J. Chem. 1990, 43, 1175.

10. Barlin, G. B.; Jiravinya, C.; Yan, J. H. Aus. J. Chem. 1991, 44, 677.

11. Edwards, M. L; Ritter, H. W.; Stemerick, D. M.; Stewart, K. T. J. Med. Chem. 1983, 26, 431.

12. Dimmock, J. R.; Kumar, P. Curr. Med. Chem. 1997, 4, 1.

13. Cardellicchio, C.; Ciccarella, G.; Naso, F.; Perna, F.; Tortorella, P. Tetrahedron 1999, 55, 14685.

14. Cardellicchio, C.; Ciccarella, G.; Naso, F.; Schingaro, E.; Scordari, F. Tetrahedron: Asymm. 1998, 9, 3667.

15. Palmieri, G. Tetrahedron: Asymm. 2003, 11, 3361.

16. Liu, D. -X.; Zhang, L. C.; Wang, Q.; Da, C. S.; Xin, Z. Q.; Wang, R.; Choi, M. C. K.; Chan. A. S. C. Org. Lett. 2001, 3, 2733.

17. Cimarelli, C.; Palmieri, G.; Volpini, E. Tetrahedron: Asymm. 2002, 13, 2417.

18. Cimarelli, C.; Mazzanti, A.; Palmieri, G.; Volpini, E. J. Org. Chem. 2001, 66, 4759.

19. Ji, J. X.; Qiu, L. Q.; Yip, C. W.; Chan, A. S. C. J. Org. Chem. 2002, 68, 1589.

20. Betti, M. Gazz. Chim. Ital. 1900, 30 II, 310.

21. Chi, K.-W.; Ahn, Y. S.; Shim, K. T.; Park, T. H.; Ahn, J. S. Bull. Korean. Chem. Soc. 1999, 20, 973.

22. Saidi, M. R.; Azizi, N.; Naimi-Jamal, M. R. Tetrahedron Lett. 2001, 42, 8111.

23. Saidi, M.; Khalaji, H. R. J. Chem. Res. (Synop.), 1997, 340.

24. Sharifi, A.; Mirzaei, M.; Naimi-Jamal, M. R. Monatsh. Chem. 2001, 132, 875. 
25. Katritzky, A. R.; Abdel-Fattah, A. A. A.; Tymoshenko, D. O.; Belyakov, S. A.; Ghiviriga, I.; Steel, P. J. J. Org. Chem. 1999, 64, 6071.

26. Paul, N.K.; Dietrich, L. M.; Jha, A. Synth. Commun. 2007, 37, 877.

27. Jha, A.; Paul, N. K.; Trikha, S.; Cameron, T. S. Can. J. Chem. 2006, 84, 843.

28. Brown, P. D. Med. Oncol. 1997, 14, 1.

29. Johnson, K. W.; Lofland, D.; Moser, H. E. Curr. Drug Targets Infect. Disord. 2005, 5, 39.

30. Lowther, W. T.; Matthews, B. W. Biochim. Biophys. Acta. 2000, 1477, 157.

31. Waller, A.S.; Clements, J. M. Curr. Opin. Drug Discov. Devel. 2002, 5, 785.

32. Chappell, L. T. Altern. Med. Rev. 1998, 3, 426.

33. Ford M.D. In Heavy Metals, 4th Ed.; McGraw Hill: New York, 1996.

34. Dallinger, D.; Kappe, C. O. Chem. Rev. 2007, 107, 2563.

35. Lidström, P.; Tierney, J.; Wathey, B.; Westman, J. Tetrahedron 2001, 57, 9225.

36. El Ashry, E. S. H.; Kassem, A. A. Arkivoc 2006, (ix), 1.

37. Menage, S.; Baley, J. B.; Dumats, J.; Hussler, G.; Seite, M.; Luneau, I. G.; Chottard, G.; Fontecave, M. J. Am. Chem. Soc. 1998, 120, 13370.

38. Szabo, J. L.; Hill, D.; Bruce, W. F. US Patent, US 2739 981, 1952. 